\title{
Physiotherapy as a first point of contact in general practice: a solution to a growing problem?
}

\author{
Rob W. Goodwin ${ }^{1}$ and Paul A. Hendrick ${ }^{2}$ \\ ${ }^{1}$ Musculoskeletal Clinics Team, Nottingham City Care, Nottingham, UK \\ ${ }^{2}$ School of Health Sciences, University of Nottingham, Nottingham, UK
}

\begin{abstract}
Aim: To evaluate the clinical effectiveness, patient satisfaction and economic efficacy of a physiotherapy service providing musculoskeletal care, as an alternative to GP care. Background: There is a growing demand on general practice resources. A novel '1st Line Physiotherapy Service' was evaluated in two GP practices (inner city practice, university practice). Physiotherapy, as a first point of contact, was provided as an alternative to GP care for patients with musculoskeletal complaints. Participants: A convenience cohort sample of over 500 patients with a musculoskeletal complaint was assessed within the physiotherapy service. For the economic evaluation a cohort of $100 \mathrm{GP}$ patients was retrospectively reviewed. Method: Clinical outcome measures were collected at assessment, one and six months following assessment. Patient satisfaction was collected at assessment. An economic evaluation was undertaken on the physiotherapy cohort of patients and compared to a retrospective cohort of patients $(n=100)$ seen by a GP. This evaluation considered only the health care perspective (primary and secondary care). Societal issues such as absence from employment were not considered. Results: There were no adverse events associated with the physiotherapy service. Patients reported high levels of satisfaction with the physiotherapy service. Patients managed within the 1st Line Physiotherapy Service demonstrated clinical improvements (EQ-5D-5L, Global Rating of Change) at the sixmonth point. There was a statistically significant difference in favour of the physiotherapy groups using a non-parametric bootstrap test; inner city practice, mean difference in costs $=£ 538.01(P=0.006 ; 95 \% \mathrm{Cl} ; £ 865.678, £ 226.98)$, university practice mean difference in costs $=£ 295.83(P=0.044 ; 95 \% \mathrm{Cl} ; £ 585.16, £ 83.69)$. Conclusion: The limitations of this pragmatic service evaluation are acknowledged. Nevertheless, the physiotherapy service appears to provide a safe and efficacious service. The service is well received by patients. There appear to be potential financial implications to the health economy. Physiotherapists, as a first point of contact for patients with musculoskeletal-related complaints, could contribute to the current challenges faced in primary care.
\end{abstract}

Key words: NHS costs; physiotherapy; primary care

Received 28 July 2015; revised 5 February 2016; accepted 15 May 2016;

first published online 6 June 2016

\section{Introduction}

A number of factors are currently aligning and potentially drawing general practice to the edge of a perfect storm. These factors include an ageing population, the subsequent increase in age-related health problems, the almost epidemic increases seen in what are essentially lifestyle-related

Correspondence to: Rob W. Goodwin, Clinical Lead Physiotherapist, MSK Clinics Team, Mary Potter Centre, Gregory Boulevard, Nottingham, NG7 5HY, UK. Email: Robert.Goodwin@nottinghamcitycare.nhs.uk

(C) Cambridge University Press 2016 
health complaints and the challenges in the training, recruitment and retention of general practitioners (GPs).

Currently, the percentage of the UK population over the age of 65 is over $17 \%$. This is compared with $15 \%$ in 1985 (Office for National Statistics, 2012). The prediction is that by 2035 this will have risen again to 23\% (Office for National Statistics, 2012).

As a consequence of this rise in population there is an anticipated rise in health conditions associated with old age. In particular arthritis and degenerative joint pains can be expected to increase significantly (Department of Health, 2006) as can a range of common musculoskeletal (MSK) disorders including back pain, shoulder pain and knee pain (Urwin et al., 1998; Picavet and Schouten, 2003). Currently, the primary burden for the first point of management of these conditions is shouldered by GPs. The prevalence of patients with musculoskeletal complaints within a GPs workload has been estimated to range from 18 to 33\% (Mallen et al., 2007; Jordan et al., 2010; Margham, 2011). For a general practice with a patient population of 10000 patients this equates to a full-time equivalent caseload.

Running in parallel to these changes in the anticipated MSK health of the nation are the acknowledged work force issues within general practice. The training and recruitment of GPs is recognised as a significant current challenge to the efficacy of general practice provision. The Royal College of General Practitioners (RCGP) predicted that up to 600 practices could face closure in 2015 because of the deepening crisis in GP recruitment and retention (Royal College of General Practitioners, 2014). These challenges to general practice, in its current guise, make it almost untenable moving further into the 21 st century.

A number of potential solutions have been proposed to ensure the survival of a free-at-pointof-contact primary care service which forms the bedrock of the National Health Service (NHS). These include developing training pathways for GPs with a special interest in MSK conditions or the transfer of first-contact care to alternative health care providers.

The arguments for the re-development of primary care services have been debated and the increased role of physiotherapy in the first line management of MSK conditions previously advocated (Foster et al., 2012). Such a move would align the primary care management of MSK problems with the core competencies of the physiotherapy profession. Furthermore, physiotherapists have demonstrated competence in extended roles (McClellan et al., 2006; Stanhope et al., 2012; Sutton et al., 2015). Good patient satisfaction has also been demonstrated where these initiatives have been implemented (Reeve and May, 2009; Kennedy et al., 2010). However, this evidence has been predominantly generated in secondary care environments.

Ludvigsson and Enthoven (2012) undertook an evaluation of physiotherapists as primary assessors of patients with MSK problems in a GP practice in Sweden. They found that the service was safe and almost all patients $(85 \%)$ ) could be managed solely by the physiotherapist. They reported good patient satisfaction and of those patients managed by the physiotherapists the majority did not return to see their GP in the following three-months with the same complaint. This compared favourably to GP care. In summary the authors reported that the use of physiotherapists as primary assessors for patients with musculoskeletal disorders was a viable alternative to GP care.

At a time when this topic is growing ever more relevant this investigation attempts to further the work of Ludvigsson and Enthoven (2012) within the NHS. This evaluation explores the implementation of a '1st Line Physiotherapy Service' which delivers first point of contact care, in a general practice setting, to patients with MSK complaints. Funding for this service evaluation was provided by the Nottingham City Clinical Commissioning Group (CCG). This funding extended to the clinical provision of the service and research time for the lead researcher and a project assistant (PA).

\section{Methods}

A prospective, evaluative design was applied to the clinical evaluation of the 1st Line Physiotherapy Service with a convenience, cohort sample recruited during the 12-months that the service was delivered.

For the economic evaluation of the physiotherapy service this same cohort was used. For the economic evaluation a retrospective, GP sample was selected at random, from the 12 months 
between January 2013 and December 2013, preceding the introduction of the 1st Line Physiotherapy Service. The patients were selected by searching under a MSK filter on the electronic records system (EMIS Web). This was undertaken by GP practice administration staff who then passed on the unique identification numbers of the cohort to the PA.

All data were collected via standardised questionnaires (physiotherapy sample) or from clinical records (GP sample). The PA role included the data collection and collation and the development of excel spread sheets for data storage and manipulation. The PA was not blinded during the data collection process.

\section{Context}

Physiotherapists, working at an advanced level and employed at band 7 level, were placed in two general practices within Nottingham City. This advanced role allowed the Physiotherapists to refer for diagnostic tests (x-ray and magnetic resonance imaging (MRI) scan) and refer to secondary care. The two physiotherapists both had over 10-years clinical experience and had undertaken Masters level modules in advanced practice skills.

The two practices differed in their patient population in that one was a traditional inner city practice and the other a university practice. Each physiotherapist provided two half-day clinics per week in their respective practice. The initial trial of the service was for a period of one-year from April 2014 to April 2015.

On contacting the practice to book an appointment, patients were offered the choice of seeing the physiotherapist, as an alternative to a GP, by the receptionist staff, if they were experiencing a MSK-related complaint. The reception staff undertook no triage duties but instead showed patients a list of 'common MSK related complaints' to highlight the type of conditions suitable for physiotherapy assessment. If patients chose to see the physiotherapist they were offered an appointment. There was an expectation, based on capacity: demand modelling before the launch of the service, that the demand for physiotherapy would exceed the capacity. As such the decision was taken to set the maximum wait for a physiotherapy appointment at 10 days.
This acknowledged the limited capacity of the service and ensured patients were seen in a timely manner, matching, as far as possible, existing GP waiting times.

Appointments were 20-min in length and patients were limited to two appointments with the physiotherapist. This was aimed at replicating normal GP care as closely as possible. If patients were felt to require on-going physiotherapy input they were referred to the main primary care physiotherapy provider at their second appointment. Within the physiotherapy assessment patients were screened for non-MSK pathology and, where appropriate, offered advice and any relevant interventions, primarily based within a self-management paradigm.

\section{Analysis}

\section{Safety and governance}

The safety of the 1st Line Physiotherapy Service was analysed retrospectively by review of incidents reported by either the physiotherapists or the general practices themselves. This was done through subjective, monthly reporting and review of electronic incident reporting systems.

\section{Descriptive outcomes}

The following descriptive measures were taken; the region and the chronicity of the complaint. Interventions provided by the physiotherapists, which included exercise prescription and advice, and any onward referrals, for diagnostic investigations or secondary care, were recorded. The outcome of the assessment, and any subsequent follow-up appointment, was also recorded. For consistency a standardised excel spread sheet for recording the data was used. Codes used for collating the descriptive data are described in Table 1 . This data were collected by the PA.

\section{Quantitative outcomes}

At assessment patients were issued with a selfcomplete questionnaire booklet with outcome measures as detailed below. This was completed independently outside the consultation room. The completion of the questionnaire booklet was voluntary. As this was a service evaluation no information was collected from those patients who

Primary Health Care Research \& Development 2016; 17: 489-502 
Table 1 Descriptive coding options for; region of pain, chronicity, intervention provided, referral/s made, and intervention outcome

\begin{tabular}{|c|c|c|c|c|}
\hline Region of pain & Chronicity & Intervention & Referral/s made & Intervention outcome \\
\hline Low back pain & $\begin{array}{l}\text { Less than four } \\
\text { weeks }\end{array}$ & $\begin{array}{l}\text { Self-management } \\
\text { advice }\end{array}$ & GP - prescription & Discharged \\
\hline Neck pain & $\begin{array}{l}\text { More than four } \\
\text { weeks }\end{array}$ & Exercise prescription & $\begin{array}{l}\text { GP - non-MSK } \\
\text { problem }\end{array}$ & Follow-up appointment booked \\
\hline Shoulder pain & & & GP - red flag & Open appointment offered \\
\hline Hip pain & & & Diagnostics - x-ray & Referred to physiotherapy \\
\hline Knee pain & & & Diagnostics - MRI & Referred to secondary care \\
\hline $\begin{array}{l}\text { Upper limb } \\
\text { other }\end{array}$ & & & Secondary care & Referred to GP - non-MSK problem \\
\hline $\begin{array}{l}\text { Lower limb } \\
\text { other }\end{array}$ & & & & $\begin{array}{l}\text { Referred to GP - medical } \\
\text { management } \\
\text { Referred to GP - red flag }\end{array}$ \\
\hline
\end{tabular}

did not agree to complete the questionnaire booklet. Clinical outcome measures were only taken for the patients managed within the 1st Line Physiotherapy Service; there was no GP clinical comparison group.

For the follow-up data (one, six months) the plan was for patients to be contacted by the PA via either telephone or email. It was immediately apparent that patients were not responding to the email system and as such this was abandoned. As a result, patients completed the questionnaires verbally, in conversation with the PA, over the telephone. No other method of contact was attempted. A period of five working days was accepted either side of the scheduled data collection points. Beyond this the data was accepted as lost to the evaluation and as such a degree of attrition was anticipated.

\section{Patient satisfaction}

Following liaison with the authors of the original Swedish study (Ludvigsson and Enthoven, 2012) an English translation of their patient satisfaction questionnaire was used.

\section{Outcome of intervention}

Two clinical outcome measures were used:

The EQ-5D-5L descriptive system (EuroQol Group, 1990) was used as a standardised measure of health status. Percentage of patients demonstrating improvement between the two time points was reported. Effect size was calculated for the change in median score for the EQ-5D-5L index. In order to determine the percentage of patients whose EQ-5D index score changed from baseline to six months (improved or deteriorated) a change score of $>0.1$ was chosen. This figure was based on the reported minimally important difference for the EQ-5D of 0.074 (range -0.011 to 0.140 ) (Walters and Brazier, 2005).

The Global Rating of Change (GROC) questionnaire (Kamper et al., 2009) is a scale designed to quantify a patient's improvement or deterioration over time. The scale asks that a person assess his or her current health status, recall that status at a previous time point, and then calculate the difference between the two.

All data were inputted onto excel spread sheets. An EQ-5D-5L excel calculator was used for the EQ-5D-5L data. This enables the EQ-5D-5L data to be easily translated into simple utility scores. These scores can be further used to demonstrate the change in an individual's quality of life, due to physiotherapy intervention. This can also be collated to show the change for a whole service or a specified population.

\section{Cost data}

Although there was no clinical comparison group costs were calculated for a GP group of patients. A retrospective cohort of 100 patients (50 from each practice) who were randomly selected from GP records and who had been seen for a primary MSK complaint were selected. These patients were selected from the 12 months 
Table 2 Descriptive demographic data of patients

\begin{tabular}{lcccc}
\hline & $\begin{array}{l}\text { Inner city practice - } \\
\text { physiotherapy }\end{array}$ & $\begin{array}{l}\text { Inner city } \\
\text { practice - GP }\end{array}$ & $\begin{array}{l}\text { University practice - } \\
\text { physiotherapy }\end{array}$ & $\begin{array}{l}\text { University } \\
\text { practice - GP }\end{array}$ \\
\hline Number of patients & 219 & 50 & 336 & 50 \\
Average age & 49.6 & 54.7 & 24.8 & 23.7 \\
Male:female & $89: 130$ & $20: 30$ & $176: 158$ & $26: 24$ \\
Chronicity & & & $126(37.5 \%)$ & \\
Less than 4 weeks & $79(36.1 \%)$ & & $210(62.5 \%)$ & $17(34 \%)$ \\
More than 4 weeks & $140(63.9 \% 0$ & & $12(4 \%)$ & $16(32 \%)$ \\
Region & $21(10 \%)$ & $2(4 \%)$ & $80(24 \%)$ & $2(4 \%)$ \\
Hip & $33(15 \%)$ & $8(16 \%)$ & $69(21 \%)$ & $5(10 \%)$ \\
Knee & $66(30 \%)$ & $18(36 \%)$ & $40(12 \%)$ & $3(6 \%)$ \\
Low back pain & $19(10 \%)$ & $5(10 \%)$ & $41(12 \%)$ & $7(14 \%)$ \\
Lower limb - other & $21(10 \%)$ & $7(14 \%)$ & $24(6 \%)$ & 1.66 \\
Neck & $37(15 \%)$ & $9(18 \%)$ & 1.09 & \\
Shoulder & $22(10 \%)$ & 2.22 & & \\
Upper limb - other & 1.22 & & & \\
Average number of & & & & \\
appointments & & & & \\
\hline
\end{tabular}

between January 2013 and December 2013, preceding the introduction of the 1st Line Physiotherapy Service. The patients were selected by searching under a MSK filter on the electronic records system (EMIS Web). This was undertaken by GP practice administration staff who then passed on the unique identification numbers of the cohort to the PA who subsequently reviewed the records and retrieved the descriptive data, as per the physiotherapy cohort, with the exception of the chronicity of the complaint.

\section{Economic analysis}

Advice was sought, throughout, from a health economist. Despite the fact that equivalence has been demonstrated in interventions by GP and physiotherapy, when comparing outcome to treatment (Scholten-Peeters et al., 2006), as this had not been proved formally within this evaluation it was not felt appropriate to undertake a cost minimisation evaluation. As such the average cost per episode of care was calculated for each group. This approach has been used elsewhere in similar cohorts of patients (Holdsworth et al., 2007). Costs per case were calculated using key data relating costs acquired from sources (Table 2). Where possible, costs were taken from 2014 figures for unit costs of health and social care (Curtis, 2014). When this document did not provide specific costs the CCG provided up to date costs for procured services. Specifically, this included an average cost per case for a secondary care referral to trauma and orthopaedic surgery based on data from 2014/ 2015. This included new outpatient activity, followup activity and procedures undertaken; both day case and inpatient. This subsequent value did not include any diagnostic referrals made in secondary care. The number of new outpatient appointments was used as a proxy measure for unique episodes of care. As a result of this calculation the average cost for a secondary care referral to trauma and orthopaedics was $£ 3085 /$ patient.

The CCG also provided the costs figures for direct access MRI scan, direct access x-ray, average cost per episode of care podiatry, average cost per episode of care acupuncture, primary care cost for blood test, primary care cost for musculoskeletal diagnostic ultrasound scan.

Physiotherapy costs were based on appointment lengths of $20 \mathrm{~min}$ at mid-point band 7 level. Any additional expenditure associated with onward referral from physiotherapy was calculated using the above figures. All key data relating costs are shown in Table 3.

Costs for GP care and physiotherapy care were calculated as an average cost per patient. This was based on the retrieved data around new appointment: follow-up appointment ratios for each service, within each practice. On average a GP at the inner city practice saw a patient 2.22 times and in the university practice 1.66 times. 
Table 3 Key data relating costs

\begin{tabular}{|c|c|}
\hline Cost element & Cost \\
\hline GP consultation (including all on-costs) & $£ 46$ \\
\hline Physiotherapy consultation (including all on-costs) & $\begin{array}{l}\text { Mid-point band 7-20 min appointment including all } \\
\text { non-pay and overheads: } f 9.04 \text { on a } 43 \text {-week service }\end{array}$ \\
\hline Direct access MRI scan & £143 \\
\hline Direct access $\mathrm{x}$-ray & £31 \\
\hline Prescription costs & No cost attributed. Actual numbers reported \\
\hline Secondary care referral & $£ 3085 /$ episode of care \\
\hline Podiatry & $£ 65.19 /$ episode of care \\
\hline Acupuncture & £305/episode of care \\
\hline Blood test & £3.03 \\
\hline Ultrasound scan & $£ 45.70$ \\
\hline GP episode of care & Inner city practice; $£ 102.12$ \\
\hline $\begin{array}{l}\text { Inner city practice - based on average of } 2.22 \\
\text { consultations per patient at } f 46 / \text { consultation } \\
\text { University practice - based on average of } 1.66 \\
\text { consultations per patient at } f 46 / \text { consultation }\end{array}$ & University practice; f76.36 \\
\hline $\begin{array}{l}\text { MSK Physiotherapy episode of care - based on existing } \\
\text { contractual assessment to follow-up ration of } 1: 1.8\end{array}$ & MSK Physiotherapy episode of care; $£ 75.94$ \\
\hline
\end{tabular}

Costs for any onward physiotherapy input were based on existing contractual assessment: followup ratio of 1:1.8 for the main physiotherapy service at a cost of $£ 75.94$.

Of importance, and relevance, is the issue of medication prescription. It was initially intended that this data would be collected and included in the economic evaluation. However, accurate prescription data was not available from the GP records to attribute costs to. Furthermore, the national average 'cost-per-GP-prescription' was felt likely to exaggerate the costs as most MSK prescription costs would be less expensive than this figure. As such the decision was made to exclude prescription costs from the economic evaluation but to report of the actual number of times prescriptions were issued for the two groups.

Costs per case were calculated, as described. Total costs for the four patient cohorts (inner city physiotherapy, inner city GP, university physiotherapy, university GP) were then calculated and the average cost per episode of care was calculated by dividing this total by the number of patients in the cohort.

Furthermore, a non-parametric bootstrap was used to obtain confidence intervals for the mean differences in cost. The mean of each of these samples was calculated, and the bias-corrected bootstrap method used to calculate $95 \%$ confidence intervals for the mean differences in cost.

\section{Results}

\section{Descriptive outcomes}

The demographic information collected from the two practices is presented in Table 4. It was clear from these demographics that the cohort of patients differed between the inner city and the university practice. As a result all outcomes will be considered separately. However, based on the data obtained the physiotherapy and GP groups of patients, at the respective practices, appear to be similar in terms of age, gender and region of MSK complaint.

The 1st Line Physiotherapy Service appears to have been safe with no adverse events reported by either of the physiotherapists or, subsequently, by either of the practices.

The physiotherapist based at the inner city practice assessed 219 patients, assessment outcome measures were obtained for 140 patients. One-month outcomes measures were obtained for 108 patients and at six-months outcome measures were obtained for 71 patients. At the university practice the figures were; assessed 336, assessment outcome measures 208, one-month outcome measures 75, six-month outcome measures 59. The majority of patients attended for a single physiotherapy consultation. In the inner city practice $78 \%$ of patients were seen once and in the university practice $92 \%$ of patients were seen once. 
Table 4 Change in EQ-5D-5L at the patients attending physiotherapy from initial consultation to six-month follow-up

\begin{tabular}{llc}
\hline Practice & Inner city practice & University practice \\
\hline & Post-pre treatment change & Post-pre treatment change \\
Median & Change in EQ-5D-5L Index & 0.08 \\
Mean & 0.10 & 0.10 \\
Standard deviation of mean & 0.13 & 0.14 \\
No. of patients & 0.27 & 59 \\
\% Patients improved & 64 & 73 \\
\% Patients not improved & 72 & 27 \\
Effect size & 28 & 1.19
\end{tabular}

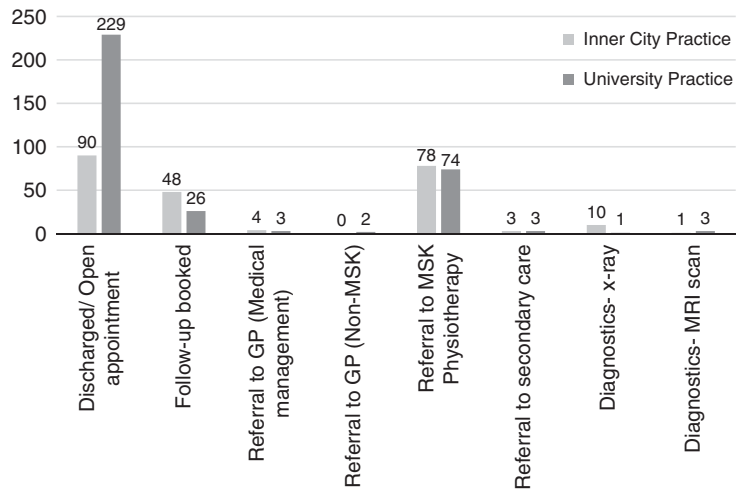

Figure 1 Resource utilisation and referral pattern of 1st Line Physiotherapy Service within inner city practice $(n=219)$ and university practice $(n=336)$

Almost all patients, regardless of practice, were offered both advice on self-management and exercise prescription on their initial assessment with the physiotherapist. Advice was provided verbally and, where necessary, patients were provided with written resources, for example exercise sheets.

Resource utilisation is reported graphically as follows (Figures 1 and 2):

In both practices the physiotherapists managed almost all of the patients independently, without recourse to a GP (university practice $99 \%$, inner city practice $98 \%$ ). This was to some extent facilitated by the extended roles they held with access to referral for diagnostics and secondary care available.

A proportion of patients were offered a followup appointment for review with the Physiotherapist within the 1st Line Service. In the inner

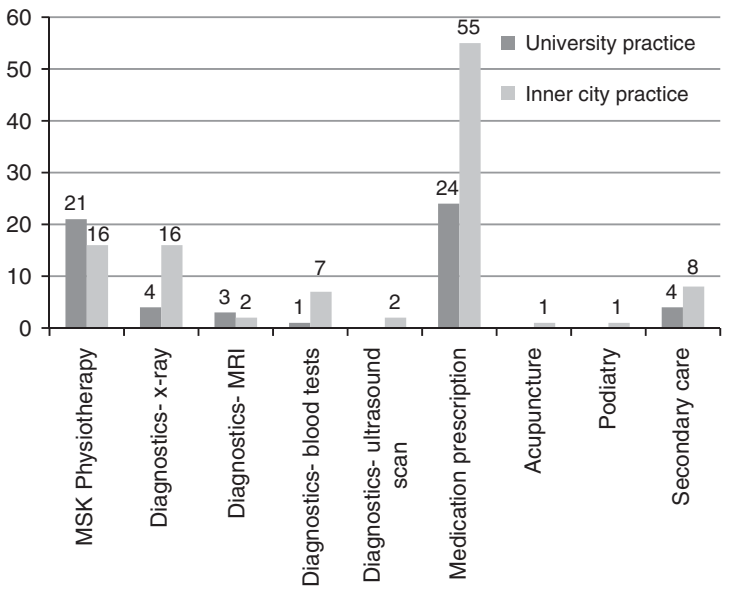

Figure 2 Resource utilisation and referral pattern of GPs within inner city practice and university practice (inner city GP $n=50$, university GP $n=50$ )

city practice there were $48(21 \%)$ follow-up appointments with 11 subsequently referred onwards to the MSK Physiotherapy Service and the remainder discharged. In the university practice there were $26(7.7 \%)$ follow-up appointments with seven patients subsequently referred onwards to the MSK Physiotherapy Service and the remainder discharged. In the university practice one patient was also referred for a MRI scan at follow-up.

Within the 1st Line Physiotherapy Service onwards referrals, excluding the MSK Physiotherapy Service, were low. Within the inner city practice onward resource utilisation was $6.4 \%$ and within the university practice onward resource utilisation was $2 \%$. 


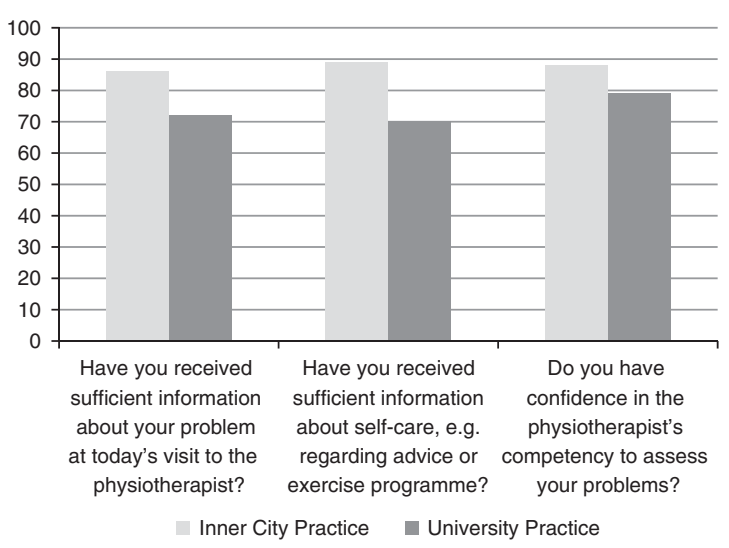

Figure 3 Patient satisfaction; percentage reporting complete satisfaction/confidence with 1st Line Physiotherapy Service

Less than $2 \%$ of patients assessed by the physiotherapist, within either practice, were referred back to see the GP for either a medical review or because they were not felt to be presenting with a MSK-related problem.

\section{Quantitative outcomes}

\section{Patient satisfaction}

The patient satisfaction questionnaire was translated into English from the original research by Ludvigsson and Enthoven (2012). Patients were asked, following their assessment with the physiotherapist, to score their response on a Likert scale, range $0-5$. There were three questions and the scores for the two practices are represented in Figure 3.

\section{Clinical outcomes}

As stated, these results are only available for the 1st Line Physiotherapy Service.

\section{Global Rating of Change (GROC)}

The GROC is designed to quantify a patient's improvement or deterioration over time. The scale asks that a person assess his or her current health status, recall that status at a previous time point, and then calculate the difference between the two. Patients at the inner city practice reported a median GROC of 0 (no different) at one-month and at the university practice the median score for the GROC was 5 (quite a bit better). Patients at the inner city practice reported a median GROC of 3 (somewhat better) at the six-month time point and at the university practice the median score for the GROC was 5 (quite a bit better).

\section{$E Q-5 D-5 L$}

EQ-5D-5L is a standardised measure of health status developed by the EuroQol Group (1990) in order to provide a generic measure of health for clinical and economic appraisal. Table 4 shows change in EQ-5D-5L, percentage of patients improved and effect size for patients attending physiotherapy at the two practices from initial consultation to six-month follow-up. As the EQ-5D-5L describes a change only patients with both pre and post scores were included in the analysis. As such the numbers are as follows; inner city practice, $n=64$, university practice, $n=59$. Effect sizes were calculated using the formula; $\mathrm{ES}=(\mathrm{M} 1-\mathrm{M} 2) / \mathrm{SD}$ where $\mathrm{M} 1$ is the assessment median score and M2 the six-month median score and SD is the standard deviation of the median assessment score (Maher and Kilmartin, 2012).

\section{Cost per average episode of care calculation}

Using the previously described key data relating to costs the following cost per average episode of care calculations were made for the two practices (Table 5). The average cost per episode of care are shown in Table 5.

The overall costs per average episode of care were significantly different between both GP practices and their respective 1st Line Physiotherapy Service equivalent. In the inner city practice the GP costs were $£ 647.16 /$ patient and the physiotherapy costs were $£ 84.26 /$ patient. In the university practice the GP costs were $£ 366.44 /$ patient and the physiotherapy costs were $£ 56.51 /$ patient.

There was a statistically significant difference in favour of the physiotherapy groups within both practices using a non-parametric bootstrap test; inner city practice, mean difference in costs = $£ 538.01(P=0.006 ; 95 \% \mathrm{CI} ; £ 865.678, £ 226.98)$, university practice mean difference in costs = $£ 295.83(P=0.044 ; 95 \% \mathrm{CI} ; £ 585.16, £ 83.69)$.

The greatest difference between the two services arose due to the differences in actual consultation costs between the two professions. With respect to resource utilisation; referrals to 
Table 5 Cost per average episode of care for service offered (GP care or 1st Line Physiotherapy care) and practice (inner city practice or university practice)

\begin{tabular}{lllll}
\hline Unit & $\begin{array}{l}\text { University practice }- \\
\text { physiotherapy }(n=336)\end{array}$ & $\begin{array}{l}\text { University practice } \\
-\mathrm{GP}(n=50)\end{array}$ & $\begin{array}{l}\text { Inner city practice }- \\
\text { physiotherapy }(n=219)\end{array}$ & $\begin{array}{l}\text { Inner city practice - } \\
\mathrm{GP}(n=50)\end{array}$ \\
\hline $\begin{array}{l}\text { Clinical cost } \\
\text { (GP consultation, }\end{array}$ & 3272.48 & 3818.00 & 2413.68 & 5106.00 \\
physio & & & & \\
consultation) & & & & \\
MRI & 429.00 & 429.00 & 143.00 & 286.00 \\
X-ray & 31.00 & 124.00 & 310.00 & 496.00 \\
Secondary care & $9,255.00$ & $123,40.00$ & $9,255.00$ & $246,80.00$ \\
Podiatry & 0 & 0 & 0 & 65.19 \\
Blood test & 0 & 16.23 & 0 & 113.61 \\
Ultrasound & 0 & 0 & 0 & 91.40 \\
Acupuncture & 0 & 0 & 0 & 305.00 \\
Physiotherapy & $5,619.56$ & $1,594.74$ & $5,923.32$ & $1,215.04$ \\
GP care & 381.80 & 0 & 408.48 & 0 \\
Total cost & $189,88.84$ & $183,21.97$ & $184,53.48$ & $323,58.24$ \\
Standard deviation & 290.75 & 902.93 & 357.88 & 1151.96 \\
Average cost per & 56.51 & 366.44 & 84.26 & 647.16 \\
episode of care & & & & \\
\hline
\end{tabular}

secondary care demonstrate a two-and-a-half fold increase seen in the inner city practice and an, almost, six-fold difference seen in the university practice. As previously noted costs for prescriptions was excluded from the cost calculations. Actual prescription activity for each practice were as follows; inner city practice (GP 55 prescriptions: physiotherapy 0 prescriptions), university practice (GP 24 prescriptions: physiotherapy 0 prescriptions).

\section{Discussion}

\section{Summary of main findings}

This service evaluation found that the 1st Line Physiotherapy Service was safe, with no adverse incidents recorded at either of the two practices. Additionally, the service appears to be well received by patients. Furthermore, within the limitations of this service evaluation, significant costs per average episode of care differences were demonstrated between usual GP care and the 1st Line Physiotherapy Service.

In the study by Ludvigsson and Enthoven (2012), of the cohort of patients who saw the physiotherapist over $80 \%$ reported complete satisfaction with the information they received from the physiotherapist and their confidence in the physiotherapists' competency to assess their problem. Both practices within this evaluation reported over $70 \%$ complete satisfaction with the same questions. This is comparable to the Swedish study generally and compares favourably to the Swedish GP cohort where satisfaction levels were closer to $50 \%$.

The number of patients that the physiotherapists managed independently compared positively to the Swedish study. Ludvigsson and Enthoven (2012) reported that, in their study, $85 \%$ of the patients did not need to be seen by a GP. Similar figures were reported in a study of physiotherapy self-referral (Holdsworth et al., 2007) in Scotland where $85 \%$ of patients needed no further referral beyond physiotherapy. The physiotherapist in the inner city practice and the university practice managed $63 \%$ and $75 \%$ of patients independently, respectively. However, this does include those, relatively few, patients who were able to make use of the physiotherapists advanced roles (x-ray, MRI scans).

Additionally, the above figures do not include those patients referred to the main MSK physiotherapy service (university practice $22 \%$, inner city practice $36 \%$ ). The criteria for management within the $1^{\text {st }}$ Line Physiotherapy Service was restricted to two appointments. It is not unreasonable to think that those patients referred 
to the main physiotherapy service could, if resources allowed, have been managed satisfactorily by those physiotherapists based in the practice itself. Certainly the figures given in the Swedish study extend beyond the two-session allowance in this evaluation to allow for management to completion of care.

The numbers referred on for either a diagnostic procedure or a secondary care opinion were $6.4 \%$ (inner city practice) and $2 \%$ (university practice). This compares favourably where, even discounting referrals to the MSK Physiotherapy Service, the rate of onward resource utilisation for the GP cohort was $33 \%$ at the inner city practice and $14 \%$ at the university practice.

The number of patients who represented with the same complaint appears to also correlate well with the work of Ludvigsson and Enthoven (2012). For the inner city practice $25 \%$ of patients re-presented in the following six months and in the university practice this figure was just 9\%. The Swedish Physiotherapy Service had a re-presentation rate of $12 \%$. However, this was in a three-month period and it would be reasonable to expect this to rise over a further three months. Furthermore, they reported $48 \%$ of patients seen by a GP as representing in the subsequent three months. This would seem to allude to greater improvements in clinical outcome for the cohort of patients managed by the physiotherapists.

Clinically the $1^{\text {st }}$ Line Physiotherapy Service appears to demonstrate good efficacy. There are self-reported improvements in both the GROC and the EQ-5D-5L.

Patients at the inner city practice reported a median GROC of 0 (no different) at one-month and at the university practice the median score for the GROC was 5 (quite a bit better). Patients at the inner city practice reported a median GROC of 3 (somewhat better) at the six-month time point and at the university practice the median score for the GROC was 5 (quite a bit better). Both these six-month scores and the rate of change in score probably reinforce the difference between the two cohorts of patients with the demographic information suggesting a younger patient population with a greater proportion of peripheral musculoskeletal complaints in the university practice.

In hypothesising about the lack of change in the inner city practice at one-month, the physiotherapists anecdotally, reported a greater degree of chronicity in the inner city practice cohort of patients. This is not reflected in the data collected (inner city practice; $<4$ weeks $36.1 \%$, $>4$ weeks $63.9 \%$ ), (university practice; $<4$ weeks $37.5 \%$, >4 weeks $62.5 \%)$. Nevertheless, this may be due more to the limited parameters of measurement. Certainly, three months is often used as delineating chronicity of MSK complaints. If this had been used it may be that the data would have reflected the clinical impression and as such accounted for the slower improvement, as might be expected for a chronic complaint, described by the GROC.

The results for the EQ-5D-5L demonstrate, of those patients providing data at baseline and six months' $(n=123)$, over $70 \%$ reported an improvement. Previous work in musculoskeletal health, albeit in surgery, have suggested effect sizes between 0.2 and $<0.5$ are considered small, 0.5 to $<0.8$ considered moderate and $>0.8$ considered large (Maher and Kilmartin, 2012). Using these parameters the effect size in the inner city practice is just below moderate (0.45) and in the university practice large (1.19).

In summary, from a clinical perspective, this evaluation appears to corroborate the work of Ludvigsson and Enthoven (2012) in that physiotherapists can safely and effectively act as first line practitioners for patients with musculoskeletal complaints.

\section{Economic evaluation}

Of particular relevance and topicality is the cost efficiency of health services. Within the limitations of a pragmatic service evaluation, this piece of work appears to intimate financial incentives for the implementation of a service providing physiotherapists as a first point of contact for patients with musculoskeletal complaints.

The cheapest of the physiotherapy services was the university practice with an average cost per episode of care of $£ 56.51 /$ patient. This is compared to $£ 366.44 /$ patient for the GP cohort in the same practice. The costs for the inner city practice were £84.26/patient and £647.16/patient for the physiotherapy package and GP package, respectively.

Clearly, a significant proportion of these savings arose due to the difference in salary between the 
physiotherapists and GPs. This saving was demonstrated despite the fact that the physiotherapy appointment time was double that of the GP time. However, this does not account for the whole picture. There were also differences demonstrated in the difference in rates of referrals for diagnostic procedures and secondary care. GP onward resource utilisation exceeded that of the physiotherapists. Of most note was the use of secondary care referrals. Over six-months GPs in the inner city practice spent $£ 493.60$ / patient on secondary care compared to $£ 42.26 /$ patient by the physiotherapist and in the university practice GPs spent $£ 246.80$ / patient on secondary care compared to $£ 27.54 /$ patient by the physiotherapist. It is tempting, and perhaps not inappropriate, to hypothesise as to the reasons, and indeed the impact, of these differences but this falls outside the remit of this evaluation. Nevertheless, there does not seem to have been an under-referral by the physiotherapists' as the majority of patients appear to have been managed within the 1st Line Physiotherapy Service itself or subsequent conservative physiotherapy management.

Previous concerns expressed with regards to the proposition of physiotherapists as first line practitioners centred on both safety of patients and the expectation of an increase in resource utilisation. This evaluation seems to reinforce previous evidence that physiotherapists, with extended roles, do not utilise resources any more than their medical colleagues and in fact less so (Carr, 2003; Rabey et al., 2009).

\section{Strengths and limitations of this study}

As an evaluation of a clinical service a pragmatic approach had to be taken and, as such, there are acknowledged weaknesses in the methodology and subsequent data generated. The primary short-coming is the lack of a comparison group. The resultant lack of clinical equivalence of difference also compromises the economic evaluation with no cost minimisation or cost effectiveness analysis possible. However, as previously stated there has been, at least, equivalence demonstrated between such services in the past (Scholten-Peeters et al., 2006) and similar physiotherapy services have demonstrated clinical efficacy (Holdsworth et al., 2007).
Further challenge could be ascribed to the economic evaluation; despite costs being attributed to any further physiotherapy intervention, beyond the $1^{\text {st }}$ Line Physiotherapy Service, these costs were not fully explored; were patients subsequently referred to secondary care, were patients subsequently referred for additional diagnostic tests? Nevertheless, these challenges could equally be ascribed to the GP cohort.

With regards to prescription costs neither of the physiotherapists were prescribers (supplementary or independent). As such any recourse to prescription medication would have been made via the GP. No recommendations for GP consultations for medication reviews were made by either Physiotherapist. The Physiotherapists described recommending patients consult with their local pharmacist with respect to over the counter medication and it would seem reasonable to hypothesise that this accounts for the absence of recourse to GPs.

Clearly, the prescribing activity is different between the physiotherapy and GP groups. As previously stated we were unable to feel confident about attributing a cost to this difference due the lack of specificity about prescriptions issued. Nevertheless, this difference somewhat results in an underestimation of the cost difference for the average cost per case.

Nonetheless, despite these limitations the evidence for the cost efficiency of a service providing physiotherapy as a first point of contact appears positive but requires further controlled, comparative studies to fully evaluate the costs differences between the two approaches.

The fact that two very different practices were used is both a strength and a weakness of this evaluation. It is acknowledged that the university practice stands outside the usual inner city practice typical for Nottingham city and as such it was felt inappropriate to combine the physiotherapy outcomes. Alternatively, the clear consistencies between the two practices reinforce the efficacy of the 1st Line Physiotherapy Service.

In addition, as only one physiotherapist, at each practice, provided the clinical input this evaluation could be seen as an analysis of their individual practice rather than physiotherapy per se. Clearly this could have been addressed by changing the therapists within the practices during the evaluation period. When balanced against the need for 
consistency within the practices a decision was made not to do this. It is also within the parameters of the pragmatic nature of the evaluation that acknowledgement is made of the non-blinding of the PA.

The issues of response/loss to both baseline and follow-up bias are also acknowledged. The pragmatic approach meant that the plan was to contact patients in the physiotherapy group either by email or over the telephone by the PA. It immediately became apparent that patients were not responding to the email contact and as such this was abandoned. As such the follow-up details, at one and six months, were all collected over the telephone. To maintain some reliability a period of five working days either side of the scheduled date was permitted but inevitably this meant patients were lost from the data set. Outcome measures for 130 patients $(23 \%$ of total physiotherapy cohort) were collected at six months. This could reasonably be said to potentially bias the sample. However, the PA sought to contact all patients as timetabled and indeed this somewhat reduces this potentiality. Again, the authors would propose addressing this through a more robust methodology.

\section{Impact}

The impact of this evaluation is potentially widespread. Clearly, one of the greatest motivations for the instigation of the 1st Line Physiotherapy Service was the potential reduction in GP burden. Of importance is the fact that the service proved to be safe for patients. Furthermore, the service was well received by patients and the clinical outcomes proved satisfactory. As such, the potential positive impact of this novel service has been shown. It has been estimated that up to $30 \%$ of a general practice caseload presents with a musculoskeletal problem. Theoretically this could also reduce the GP burden.

There is also potential impact for physiotherapy and physiotherapists with greater skill development and professional autonomy. Physiotherapists continue to push back their traditional boundaries and in this evaluation the Physiotherapists were able to make referrals to secondary care and for some diagnostics (x-ray, MRI scan). Clearly, physiotherapy scope has extended elsewhere to include further diagnostic referrals, injection therapy and independent prescribing.
Hypothetically, this has the potential of further reducing GP burden.

Another finding of this evaluation is the potential cost implications of implementing a $1^{\text {st }}$ Line Physiotherapy Service. Whilst acknowledging the pragmatic nature of this service evaluation the economic analysis demonstrates encouraging results.

\section{Future research}

There are acknowledged short-comings of this pragmatic service evaluation. This clearly leaves opportunities for future research. Of fundamental importance is a randomised comparative study between GP and physiotherapy care. Not only would this validate, or otherwise, the clinical findings of this evaluation but it would also allow for a more robust economic evaluation.

There are also potentially interesting societal issues that could be explored. Anecdotal evidence from the evaluation demonstrates potential barriers to the implementation of a novel service like $1^{\text {st }}$ Line Physiotherapy. Further research into these barriers would seem to be important if the traditional model of health care delivery, in the NHS, is to be successfully modified. Certainly, this challenge appears to be necessary due to the rising demands on an increasingly unsustainable service.

\section{Conclusion}

Based on the average cost per episode of care evaluation and the clinical evaluation undertaken the $1^{\text {st }}$ Line Physiotherapy Service appears to offer a safe, clinically efficacious and financially expedient service for patients with musculoskeletal complaints in primary care. This would appear to offer a part-solution to the rising clinical and financial pressures currently encountered in primary care.

It is acknowledged that this is an area of little research and it would be useful to undertake a more controlled, comparative trial.

\section{Acknowledgements}

The support of Daniel Hammersley, Practice Manager and Linda Lawton, Practice Manager is 
acknowledged. The support of Pete Petra is acknowledged. The support of Simon Oliver, Commissioning Manager, Nottingham City Clinical Commissioning Group is acknowledged.

\section{Financial Support}

This work was supported by the Nottingham City Clinical Commissioning Group.

\section{Conflicts of Interest}

None.

\section{References}

Carr, A.J. 2003: Orthopaedic outpatient departments: an evaluation of appropriateness, effectiveness, cost effectiveness and patient satisfaction associated with the assessment and management of defined referrals by physiotherapists. London: Department of Health.

Curtis, L. 2014: Unit Costs of Health and Social Care 2014. Kent, UK.

Department of Health. 2006: The musculoskeletal services framework - a joint responsibility: doing it differently. London: Department of Health.

EuroQol Group. 1990: EuroQol - a new facility for the measurement of health-related quality of life. Health Policy 16, 199-208.

Foster, N.E., Hartvigsen, J. and Croft, P.R. 2012: Taking responsibility for the early assessment and treatment of patients with musculoskeletal pain: a review and critical analysis. Arthritis Research \& Therapy $14,205$.

Holdsworth, L.K., Webster, V.S. and McFadyen, A.K. 2007: What are the costs to NHS Scotland of self-referral to physiotherapy? Results of a national trial. Physiotherapy 93, 3-11.

Jordan, K.P., Kadam, U.T., Hayward, R., Porcheret, R., Young, C. and Croft, P. 2010: Consultation prevalence of regional musculoskeletal problems in primary are: an observational study. BMC Musculoskeletal Disorders $11,144$.

Kamper, S.J., Maher, C.G. and Mackay, G. 2009: Global rating of change scales: a review of strengths and weaknesses and considerations for design. Journal of Manual \& Manipulative Therapy 17, 163-70.

Kennedy, D.M., Robarts, S. and Woodhouse, L. 2010: Patients are satisfied with advanced practice physiotherapists in a role traditionally performed by orthopaedic surgeons. Physiotherapy Canada 62, 298-305.
Ludvigsson, M.L. and Enthoven, P. 2012: Evaluation of physiotherapists as primary assessors of patients with musculoskeletal disorders seeking primary health care. Physiotherapy 98, 131-37.

Maher, A.J. and Kilmartin, T.E. 2012: An analysis of Euroqol EQ-5D and Manchester Oxford Foot Questionnaire scores six months following podiatric surgery. Journal of Foot and Ankle Research 5, 17.

Mallen, C.D., Peat, G., Thomas, E., Dunn, K.M. and Croft, P.R. 2007: Prognostic factors for musculoskeletal pain in primary care: a systematic review. British Journal of General Practice 57, 655-61.

Margham, T. 2011: Musculoskeletal disorders: time for joint action in primary care. British Journal of General Practice 61, 657-58.

McClellan, C.M., Greenwood, R. and Benger, J.R. 2006: Effect of an extended scope physiotherapy service on patient satisfaction and the outcome of soft tissue injuries in an adult emergency department. Emergency Medicine Journal 23, 384-87.

Office for National Statistics. 2012: Population Ageing in the United Kingdom, its Constituent Countries and the European Union [online]. Retrieved 10 June 2015 from http://www.ons.gov.uk/ons/dcp171776_258607.pdf.

Picavet, H.S. and Schouten, J.S. 2003: Musculoskeletal pain in the Netherlands: prevalences, consequences and risk groups, the DMC3-study. Pain 102, 167-78.

Rabey, M., Morgans, S. and Barrett, C. 2009: Orthopaedic physiotherapy practitioners: surgical and radiological referral rates. Clinical Governance: An International Journal $14,15-19$.

Reeve, S. and May, S. 2009: Exploration of patients' perspectives of quality within an extended scope physiotherapists' spinal screening service. Physiotherapy Theory and Practice $25,533-43$.

Royal College of General Practitioners. 2014: Over 500 surgeries at risk of closure as GP workforce crisis deepens [online]. Retrieved 10 June 2015 from http://www. rcgp.org.uk/news/2014/october/over-500-surgeries-at-risk-ofclosure-as-gp-workforce-crisis-deepens.aspx.

Scholten-Peeters, G.G.M., Neeleman-van der Steen, C.W.M., van der Windt, D.A.W.M., Hendriks, E.J.M., Verhagen, A.P. and Oostendorp, R.A.B. 2006: Education by general practitioners or education and exercises by physiotherapists for patients with whiplash-associated disorders? A randomized clinical trial. Spine 31, 723-31.

Stanhope, J., Grimmer-Somers, K., Milanese, S., Kumar, S. and Morriss, J. 2012: Extended scope physiotherapy roles for orthopaedic outpatients: an update systematic review of the literature. Journal of Multidisciplinary Healthcare 5, 37-45.

Sutton, M., Govier, A., Prince, S. and Morphett, M. 2015: Primary-contact physiotherapists manage a minor trauma caseload in the emergency department without misdiagnoses or adverse events: an observational study. Journal of Physiotherapy 61, 77-80. 
Urwin, M., Symmons, D., Allison, T., Brammah, T., Busby, H., Roxby, M., Simmons, A. and Williams, G. 1998: Estimating the burden of musculoskeletal disorders in the community: the comparative prevalence of symptoms at different anatomical sites, and the relation to social deprivation. Annals of the Rheumatic Diseases 57, 649-55.

Walters, S.J. and Brazier, J.E. 2005: Comparison of the minimally important difference for two health state utility measures: EQ-5D and SF-6D. Quality of Life Research 14, 1523-532. 